\title{
Structural plasticity of the Salmonella FliS flagellar export chaperone
}

Ráchel Sajó ${ }^{1}$, Orsolya Tőke ${ }^{2}$, István Hajdú ${ }^{1}$, Hajnalka Jankovics ${ }^{3}$, András Micsonai $^{4}$, József Dobó ${ }^{1}$, József Kardos ${ }^{4}$, Ferenc Vonderviszt ${ }^{3,5, *}$

${ }^{1}$ Institute of Enzymology, Research Centre for Natural Sciences, Hungarian Academy of Sciences, Magyar Tudósok krt. 2, H-1117 Budapest, Hungary

${ }^{2}$ Institute of Organic Chemistry, Research Centre for Natural Sciences, Hungarian Academy of Sciences, Magyar Tudósok krt. 2, H-1117 Budapest, Hungary

${ }^{3}$ Bio-Nanosystems Laboratory, Research Institute for Chemical and Process Engineering, University of Pannonia, Egyetem u. 10, H-8200 Veszprém, Hungary ${ }^{4}$ MTA-ELTE NAP B Neuroimmunology Research Group, Department of Biochemistry, Institute of Biology, Eötvös Loránd University, Pázmány Péter sétány 1/C, H-1117 Budapest, Hungary

${ }^{5}$ Institute of Technical Physics and Materials Science, Centre for Energy Research, KonkolyThege u. 29-33, H-1121 Budapest, Hungary 


\begin{abstract}
The Salmonella FliS flagellar export chaperone is a highly $\alpha$-helical protein. Proteolytic experiments suggest that FliS has a compact core. However, the calorimetric melting profile of FliS does not show any melting transition in the $25-110^{\circ} \mathrm{C}$ temperature range. $\mathrm{CD}$ measurements reveal that FliS is losing its helical structure over a broad temperature range upon heating. These observations indicate that FliS unfolds in a non-cooperative way and its native state shows features reminiscent of the molten globule state of proteins possessing substantial structural plasticity. As FliS has several binding partners within the cell, conformational adaptability seems to be an essential requirement to fulfill its multiple roles.
\end{abstract}

\title{
Keywords
}

Flagellar export chaperone; FliS; flagellar export system; non-cooperative unfolding; atypical molten globule

\author{
Abbreviations \\ CD, circular dichroism; DSC, differential scanning calorimetry; NMR, nuclear magnetic \\ resonance; MD, molecular dynamics; MG, molten globule; FliC, flagellin; TFE, 2,2,2- \\ trifluoroethanol
}

\section{Highlights}

- The FliS flagellar export chaperone is an antiparallel four-helix-bundle protein.

- Salmonella FliS does not show cooperative melting transition.

- The native state of FliS exhibits molten-globule-like features.

- Non-cooperative unfolding suggests structural plasticity essential for function.

*Corresponding author, email: von007@almos.vein.hu 


\section{Introduction}

Flagella are locomotion organelles of the bacterial cells. The flagellum consists of three main parts: the basal body which is embedded into the cell membrane, the long helical filament and the flexible hook, that connects the basal body and the filament. External flagellar proteins are synthesized in the cytoplasm and translocated to the distal end of the growing structure by a specific type-III secretion apparatus [1]. A narrow ( 2 $\mathrm{nm}$ wide) central channel spans the whole flagellum through which the flagellar axial proteins are transported to their site of polymerization. Premature assembly of flagellar structural proteins must be prevented in the cytoplasm. The substrate specific flagellar chaperones are believed to play this role within the cell [2-4]. The main function of the chaperones is to maintain their cognate substrates in the secretion-competent monomeric form and promote their delivery to the gate of flagellar export machinery [5-9].

FliS is the specific chaperone for the major flagellar filament component protein, flagellin (FliC) $[2,10,11]$. It is a small protein, consisting of about 135 amino acids, which has multiple functions within the flagellar export system. Besides facilitating flagellin export it has been demonstrated that FliS also interacts with other flagella-related proteins [12-14] and plays a role in the transcriptional regulation of flagellar biosynthesis, too [15-17].

The X-ray structure of FliS from Aquifex aeolicus revealed that the protein contains an antiparallel four-helix-bundle with a quasi-helical cap formed by $16 \mathrm{~N}$-terminal amino acid residues [18]. The crystal structures of Helicobacter pylori and Bacillus subtilis FliS also show a highly helical structure [12,19]. The 3D structure of the $14.7 \mathrm{kDa}$ Salmonella typhimurium FliS has not been determined yet but suggested to be predominantly $\alpha$-helical as well $[2,16]$. Proteins with a framework consisting of a pair of antiparallel helical bundles typically show high thermal stability and a clear two-state folding/unfolding behavior [20]. In contrast, our results demonstrate that Salmonella FliS does not show a cooperative melting transition and exhibits features reminiscent of molten globular proteins. 


\section{Materials and Methods}

\section{Protein expression and purification}

His $_{6}$-tagged FliS was expressed in BL21(DE3)pLysS cells (Novagen-Merck), and purified as described previously [21]. FliC monomers were purified as described by Vonderviszt et al. [22]. Protein concentrations were determined from absorption measurements at $280 \mathrm{~nm}$ using molar extinction coefficients $\left(\varepsilon_{280}=17880 \mathrm{M}^{-1} \mathrm{~cm}^{-1}\right.$ for flagellin and $\varepsilon_{280}=11460 \mathrm{M}^{-1} \mathrm{~cm}^{-1}$ for FliS) calculated from the known aromatic amino acid contents of the molecules [23]. Purity of protein samples was checked using SDS-PAGE followed by Coomassie blue R-250 staining.

\section{Homology modeling and molecular dynamics simulations}

The protein sequence of Salmonella typhimurium FliS without the His ${ }_{6}$-tag was uploaded to the IntFOLD Server, Version 2.0 [24]. We have chosen the homology model from the top 5 models, which were based on the structures of Helicobacter pylori (Lam et al. [12], PDB: 3IQC) and Aquifex aeolicus FliS (Evdokimov et al. [18], PDB: 1ORJ). The homology model was subjected to molecular dynamics (MD) simulation as implemented in GROMACS [25], using the AMBER-ff99SB*-ILDNP force field [26]. The system was solvated by approximately 10000 water molecules with TIP4P parametrization [27]. The total charge of the system was neutralized, and the physiological salt concentration was set by placing $\mathrm{Na}^{+}$ and $\mathrm{Cl}^{-}$ions. Energy minimization of starting structures was followed by sequential relaxation of constraints on protein atoms in three steps and an additional NVT step (all of 200 ps) to stabilize pressure. Trajectories of subsequent $150 \mathrm{~ns}$ NPT simulations at $300 \mathrm{~K}, 373 \mathrm{~K}$ and $300 \mathrm{~K}$ at 1 bar were recorded (collecting snapshots at every $20 \mathrm{ps}$ ). The molecule reached equilibrium conformation in $30 \mathrm{~ns}$ in the simulations. For comparison, A. aeolicus FliS X-ray structure (1ORJ) and globular helical proteins such as lysozyme (193L) and human growth hormone (1HGU) were also subjected to MD simulations. Secondary structure of the frames of MD simulation was determined by the DSSP algorithm [28]. Flexibility of the structure at the residue level was evaluated by the root mean square fluctuation (RMSF) which is the square root of the variance of the fluctuation around the average position of the backbone [25]. Molecular graphics was performed with the UCSF Chimera package (University of California, San Francisco) supported by NIGMS P41-GM103311 [29]. 


\section{CD and fluorescence measurements}

All samples were dialyzed against $10 \mathrm{mM}$ Na-phosphate buffer ( $\mathrm{pH}$ 7.4). CD measurements were performed in sealable quartz cells of $1 \mathrm{~mm}$ pathlength using a Jasco J-810 (Jasco, Tokyo, Japan) spectropolarimeter. Far-UV CD spectra were recorded in the range of 185-260 nm between 5 and $110{ }^{\circ} \mathrm{C}$ in $7{ }^{\circ} \mathrm{C}$ steps. A continuous heat-up of the sample was recorded at $192 \mathrm{~nm}$ from $5{ }^{\circ} \mathrm{C}$ to $110{ }^{\circ} \mathrm{C}$ with scanning rates of $120{ }^{\circ} \mathrm{C} /$ hour or $40{ }^{\circ} \mathrm{C} /$ hour. Temperature was controlled using a PTE Peltier unit. The thermal denaturation profile was fitted according to the Gibbs-Helmholtz equation assuming a two-state model, which is represented by a sigmoidal curve [30]. Helical content as a function of TFE concentration was investigated by Synchrotron Radiation (SR) CD at DISCO beamline at Synchrotron SOLEIL at $0.4 \mathrm{mg} / \mathrm{ml}$ (26 $\mu \mathrm{M})$ concentration in a $\mathrm{CaF}_{2}$ cell of $40 \mu \mathrm{m}$ pathlength in the range of 185-270 $\mathrm{nm}$. CD spectra were quantitatively analyzed by the recently developed BeStSel method [31]. Similarly to some other algorithms, BeStSel makes a distinction between the regular, middle part of $\alpha$-helices (helix 1), where all the backbone H-bonds are formed, and the spectrally different ends of the $\alpha$-helices (two-two residues at both ends, named helix 2). Helix 2 content and the ratio of the two components can be used to estimate the number and average length of $\alpha$-helices.

Intrinsic fluorescence experiments were carried out on a Jobin Yvon FluoroMax-3 (Horiba Jobin Yvon, Edison, NJ) spectrofluorimeter at $25^{\circ} \mathrm{C}$. Emission spectra were recorded in the range of $300-400 \mathrm{~nm}$ by selective excitation of Trp fluorescence at $297 \mathrm{~nm}$. Emission/excitation slits of $5 / 5 \mathrm{~nm}$ were used. FliS, FliC or the FliS:FliC complex were measured at $6.5 \mu \mathrm{M}$ concentration for each protein. Heat-induced changes were measured in the temperature range of $20-90{ }^{\circ} \mathrm{C}$ by thermostating the samples by the built-in Peltier element of the fluorimeter.

\section{Differential scanning calorimetry (DSC)}

DSC experiments were performed with a VP-DSC instrument (MicroCal, Northampton, MA) with a scanning rate of $1^{\circ} \mathrm{C} / \mathrm{min}$ in the range of $25-110^{\circ} \mathrm{C}$ using a protein concentration of $0.34 \mathrm{mg} / \mathrm{ml}$ for both FliS and FliC. The samples were extensively dialyzed against $10 \mathrm{mM}$ Na-phosphate buffer ( $\mathrm{pH}$ 7.4). The dialysis buffer was used as reference during the measurements. Data were analyzed with the Origin 5.0-based MicroCal software package. Both after the DSC and the CD measurements the samples were analyzed by SDS-PAGE to verify that no degradation occurred during the experiment. 


\section{Limited proteolysis}

Limited proteolysis by trypsin and subtilisin was performed in $20 \mathrm{mM} \mathrm{Na} / \mathrm{K}$-phosphate buffer, $\mathrm{pH} 7.8$, containing $300 \mathrm{mM} \mathrm{NaCl}$ at room temperature at a FliS concentration of 0.4 $\mathrm{mg} / \mathrm{ml}$. In a typical digestion experiment the protease was added to the protein solution at a 1:300 (w/w) ratio. At various time points, $20 \mu \mathrm{l}$ samples were taken and mixed with $20 \mu \mathrm{l}$ electrophoretic sample buffer, containing $0.3 \mathrm{mg} / \mathrm{ml}$ TLCK or PMSF protease inhibitor, followed immediately by heating in a boiling water bath for $10 \mathrm{~min}$. Electrophoresis was performed on $15 \%$ polyacrylamide slab gels. Bands were sliced out and were subjected to exhaustive in-gel digestion by trypsin. The extracted peptides were subjected to LC-MS/MS analysis to identify the corresponding FliS fragment.

\section{NMR spectroscopy}

One-dimensional ${ }^{1} \mathrm{H}$ NMR experiments were carried out on a Varian NMR System $(600 \mathrm{MHz}$ for ${ }^{1} \mathrm{H}$ ) (Varian Inc., Palo Alto CA) five-channel spectrometer using a 5-mm indirect detection triple resonance $\left({ }^{1} \mathrm{H}^{13} \mathrm{C}^{15} \mathrm{~N}\right) \mathrm{z}$-axis gradient probe. Solvent water suppression was achieved by presaturation. Measurements were done at $0.5 \mathrm{mg} / \mathrm{ml}$ protein concentration in $10 \mathrm{mM} \mathrm{Na}$ phosphate buffer $(\mathrm{pH} 7.4)$ at $25^{\circ} \mathrm{C}$. FliS was prone to aggregation at higher concentrations. 


\section{Results}

\section{Temperature-induced unfolding of FliS}

Heat denaturation of Salmonella FliS was monitored by differential scanning calorimetry. For comparison, the melting profile of flagellin, the main binding partner of FliS, was also measured. While the thermal unfolding curve of FliC exhibited a sharp peak around $48^{\circ} \mathrm{C}$, FliS did not show any cooperative melting transition in the range of $25-110^{\circ} \mathrm{C}$ and the obtained calorimetric curve did not contain any peak (Fig. 1). This behavior is characteristic for intrinsically disordered proteins or proteins in molten globule state.

Available structural information suggests that FliS is a highly $\alpha$-helical protein $[16,18]$. The thermal behavior of FliS was also investigated by circular dichroism spectroscopy in the far UV region which provides information about the secondary structure of proteins. Far-UV CD spectra recorded between $5^{\circ} \mathrm{C}$ and $110^{\circ} \mathrm{C}$ are presented on Figure $2 \mathrm{~A}$. Heat denaturation of FliS was almost completely reversible under the applied conditions. Close to room temperature, the CD spectrum of FliS is characteristic of a protein largely dominated by $\alpha$ helical structure. Upon heating, the CD signal is gradually decreasing over a wide range of temperature. Analysis of the $\mathrm{CD}$ spectra by the BeStSel method [31] reveals that at $110{ }^{\circ} \mathrm{C}$ the proportion of $\alpha$-helical segments is reduced drastically to about $20 \%$ of the original amount. The measured spectra have an isodichroic point at $203 \mathrm{~nm}$, which is a typical characteristic of two-state transitions. However, detailed analysis of the secondary structure by the BeStSel algorithm shows that with increasing temperature, the length of $\alpha$-helices starts to decrease above $40{ }^{\circ} \mathrm{C}$, while the number of helices decrease only above $60{ }^{\circ} \mathrm{C}$, which is contradictory to the two-state system model (Fig. 2C). We assume that in this case the two distinct states are not at the overall tertiary structural level corresponding to the native and unfolded conformations, instead we observe an $\alpha$-helix to coil transition at the level of the individual helices as the temperature increases.

Conformational changes were also monitored at a fixed wavelength of $192 \mathrm{~nm}$ at heating rates of 120 and $40^{\circ} \mathrm{C} /$ hour (Fig. 2C). In consistence with the calorimetric results, the heat-up profile does not show a well-defined narrow transition. The $\alpha$-helical structure is lost continuously and the most significant changes occur between $40-100^{\circ} \mathrm{C}$, which is an unusually broad temperature range for heat induced unfolding. The measured curve has a shape of a broad sigmoidal and can be fitted by a cooperative two-state model [30] providing values of $70-80 \mathrm{~kJ} / \mathrm{mol}$ and $72^{\circ} \mathrm{C}$ for the enthalpy change $(\Delta H)$ and melting temperature $\left(T_{\mathrm{m}}\right)$, 
respectively. The fitting was acceptable when $\Delta C_{p}$ of unfolding was set to $0-1 \mathrm{~kJ} \mathrm{~K}^{-1} \mathrm{~mol}^{-1}$. $\Delta C_{p}$ of unfolding of well ordered, stable globular proteins is reported to be $\sim 50 \mathrm{~J} / \mathrm{mol}$ per residue resulting mainly from the hydration effect of nonpolar side chains upon unfolding [32]. A globular protein with the size of FliS should have a $\Delta C_{p}$ of $\sim 7 \mathrm{~kJ} / \mathrm{mol}$, in contrast to the observed nearly zero $\Delta C_{p}$. The obtained $\Delta H$ is also very small as compared to values typical for globular proteins of similar sizes. As examples, native $\beta_{2}$-microglobulin, a globular protein of $12 \mathrm{kDa}$, exhibits an enthalpy change of $340 \mathrm{~kJ} / \mathrm{mol}$ upon heat denaturation around $65^{\circ} \mathrm{C}$ [33]. Rop, a four-helix-bundle protein having a protein fold similar to FliS, exhibits 550 $\mathrm{kJ} / \mathrm{mol}$ enthalpy change with a midpoint of $\sim 70{ }^{\circ} \mathrm{C}$ upon denaturation [34]. We suggest that the very wide range of transition and the exceptionally small $\Delta H$ and $\Delta C_{p}$ might be consistent with a gradual unfolding the FliS protein losing its ordered structure in a non-cooperative way.

Intrinsic fluorescence measurements were applied to reveal heat-induced structural changes in the local environment the lone Trp residue (W122) of FliS. The wavelength of the maximal fluorescence intensity, which reflects the hydrophobicity of the environment of the indole ring of tryptophan, was red-shifted from $342 \mathrm{~nm}$ with increasing temperature. The 355/342 nm fluorescence intensity ratio (Fig. 2D) reveals two phases of conformational changes suggesting that heat-induced conformational rearrangements within the vicinity of W122 follow a different kinetics as compared to the overall structural changes shown by far-UV CD (Fig. 2C).

\section{Characterization of the functional state of FliS}

Thermal behavior of Salmonella FliS exhibited unusual features. It was essential to demonstrate that FliS was in a functional state under the applied conditions. Isothermal titration calorimetry was used to judge that FliS was able to recognize and bind its major partner, flagellin, with a micromolar dissociation constant as was reported previously [11]. Fluorescence measurements also confirmed the interaction between FliS and FliC. Addition of FliC to the chaperone resulted in spectral changes of the intrinsic fluorescence of the single tryptophan residue W122 of FliS (Fig. 3A). The fluorescence emission spectrum of FliS has a maximum at $341 \mathrm{~nm}$, however, upon complex formation with FliC the maximum shifted to $329 \mathrm{~nm}$ and the fluorescence intensity significantly increased. These observations clearly show that under our experimental conditions FliS is capable of binding FliC. 
The structural integrity of FliS was further evidenced by one-dimensional ${ }^{1} \mathrm{H}$ NMR measurements performed at room temperature (Fig. 3B). Chemical shifts in both the aromatic/amide proton and the aliphatic regions show a significant dispersion characteristic of folded proteins. Specifically, downfield-shifted amide protons $\left(\delta_{\mathrm{HN}}>8.5 \mathrm{ppm}\right)$ and methyl proton resonances affected by ring currents near and below $0 \mathrm{ppm}$ indicate an ordered tertiary structure. Further, the line width of the resolved $\mathrm{NH}$ side chain resonance of W122 corresponds to that expected for a folded $15 \mathrm{kDa}$ protein.

Proteolytic experiments also confirmed that FliS has a compact core part. When FliS was digested by trypsin or subtilisin, two small terminal segments were very quickly removed by the proteases in the early phase of digestion but the resulting roughly $12 \mathrm{kDa}$ fragment displayed significant resistance against further degradation (Fig. 4). When flagellin was digested under similar conditions [22], its disordered terminal regions were fully degraded within a few minutes, and its F40 compact fragment was slowly digested further on a similar time scale. The very similar digestion patterns obtained by two different proteases indicate that Salmonella FliS has a folded core. Mass spectrometry analysis revealed that the $12 \mathrm{kDa}$ metastable fragment lacked $14 \mathrm{NH}_{2}$-terminal residues (including the $\mathrm{His}_{6}$-tag) and 7 residues from the C-terminal end of the polypeptide chain. The easily degraded terminal segments do not belong to the conserved helical regions of FliS. They seem to be highly mobile and unstructured as suggested by MD simulations (Fig. 5B). The corresponding segments are often not visible in the electron density maps available for FliS molecules from other bacterial sources.

\section{Dynamic secondary structure of FliS}

The 3D structure of FliS from Salmonella typhimurium has not been experimentally determined yet. The structures of Aquifex aeolicus, Helicobacter pylori and Bacillus subtilis FliS proteins have been determined by X-ray crystallography, revealing that all these proteins consist of an antiparallel four-helix bundle $[12,18,19]$. There is sequence homology of 28,35 , and $34 \%$ identity between Salmonella and A. aeolicus, H. pylori and B. subtilis FliS molecules, respectively. A homology model was constructed by the IntFOLD Server [24] for Salmonella FliS. Because the initial model showed sterical clashes, it was equipped with hydrogens, solvated with water, and subjected to energy minimization, and $150 \mathrm{~ns} \mathrm{MD}$ simulations in explicit solvent. First, a simulation at $300 \mathrm{~K}$ was carried out, which was followed by a heat treatment at $373 \mathrm{~K}$ and a final simulation at $300 \mathrm{~K}$. An equilibrium was 
reached in the first $30 \mathrm{~ns}$ in each simulation. Interestingly, in the first $30 \mathrm{~ns}$ of the simulation at $373 \mathrm{~K}$, the four helices were rearranged forming a more compact core with helices oriented more parallel with each other. This conformation was stable during the simulation and was also preserved under a final MD simulation at $300 \mathrm{~K}$ (Fig. 5A). The flexible, fluctuating regions of the FliS molecule were determined from the RMSF values of the MD trajectory revealing that mainly the $\mathrm{N}$ - and $\mathrm{C}$-terminal parts of the molecule exhibit high flexibility (Fig. 5B). In the final simulation step, the helix content of the molecule was fluctuating between 46 and 67\% with a maximal frequency at 57\% (Fig. 5C). For reference, Aquifex FliS and globular helical proteins exhibiting cooperative unfolding transition around $70^{\circ} \mathrm{C}$, such as lysozyme (PDB:193L [30]) and human growth hormone (PDB:1HGU [35]) were also subjected to MD simulations. The helix content of Salmonella and Aquifex FliS molecules fluctuated significantly more resulting in a broader distribution profile than the cooperatively folded control proteins (Fig. 5C). A sharp profile with a half width of $\sim 3 \%$ was found for the helix fluctuations in MD simulation of the cooperatively folded myoglobin [36].

In accordance with the model calculations, the far-UV CD spectrum of Salmonella FliS obtained at room temperature is characteristic of a predominantly $\alpha$-helical protein (Fig. 2, Fig. 5D). Analysis of the measured CD spectrum by the BeStSel method [31] estimated 51\% $\alpha$-helical content, which, taking into account the presence of the disordered $\mathrm{His}_{6}$-tag, agrees well with the model. However, this value is lower than expected compared to the available Xray structures of FliS molecules from the above mentioned three organisms exhibiting $\alpha$-helix contents in the range of $62.5-70.2 \%$ as determined by the DSSP algorithm [28]. This discrepancy was investigated by applying 2,2,2-trifluoroethanol (TFE) which is known to promote hydrogen bonding. TFE at low or moderate concentrations $(<30 \mathrm{v} / \mathrm{v} \%)$ stabilizes helices only in regions that have a high intrinsic helical propensity [37,38]. The effect of TFE on the secondary structure formation in FliS was studied by SRCD in the 185-250 $\mathrm{nm}$ region (Fig. 5D). Addition of TFE resulted in the increase of $\alpha$-helical signal. At 30\% TFE, FliS exhibited $74 \% \alpha$-helical structure which is similar to the values found for the available X-ray structures. Our TFE studies indicate that FliS is not completely folded at room temperature in solution. 


\section{Discussion}

The FliS flagellar chaperone from Salmonella is a highly $\alpha$-helical protein as suggested by homology modeling and CD measurements. Proteolytic experiments indicated that it has a compact core part. However, scanning calorimetric measurements revealed that FliS does not show a cooperative melting transition. Monitoring the heat-induced structural changes by $\mathrm{CD}$ spectroscopy demonstrated that the ordered secondary structure is lost over a very broad temperature range $\left(40-100^{\circ} \mathrm{C}\right)$ which may be indicative of gradual unfolding of the native structure. This was further supported by the analysis of the measured CD spectra which revealed that temperature increase resulted first in the shortening of helices and their number decreased only at higher temperatures (Fig. 2). MD simulations showed relatively large fluctuations in the $\alpha$-helix content compared to that of stable proteins exhibiting cooperative unfolding transitions with similar temperature midpoints (Fig. 5C). Aquifex FliS showed a similar broad profile indicating that it might be a general property of FliS molecules from various sources. Fluorescence monitoring of the local environment of the lone W122 residue also showed a somewhat different unfolding behavior as compared to the overall characteristics revealed by CD spectroscopy. These observations suggest that various parts of the molecule unfold in a non-cooperative manner.

Substantial amount of secondary structure but lack of cooperativity in unfolding are typical characteristics of the molten globule (MG) state of proteins [39]. According to the classical view, proteins in the MG state are associated with functional inactivity, but FliS can strongly bind its cognate partner FliC as confirmed by ITC and fluorescence measurements. Recent studies have revealed, however, that the MG state does not necessarily preclude efficient catalysis $[40,41]$ or specific molecular recognition functionality $[42,43]$. MGs are believed to have fluctuating tertiary structure, while our NMR measurements indicated a folded core for FliS. A similar situation was observed for the MG state of nuclear coactivator binding domain (NCBD) of CREB binding protein, which contains a small well-folded core but lacks cooperative unfolding behavior [42]. The internal dynamics of NCBD is caused by interconversion between discrete folded conformations. Despite populating compact folded conformations, NCBD still has a slowly fluctuating tertiary structure and thus adheres to the definition of a MG. Recent studies have also shown slow exchange between several well defined conformations in the MG state of $\alpha$-lactalbumin, representing dynamic ensemble states with specific tertiary interactions [44]. FliS may also readily assume different conformations as indicated by the structural heterogeneity of subunits even within the unit cell 
of the available Aquifex FliS crystal structure (Fig. 6A) [15]. Badger et al. determined the Xray structure of Bacillus subtilis FliS [19], which also demonstrates the structural plasticity of the molecule because it forms a dimer of four-helix-bundles in a domain swapped manner in the unit cell (Fig. 6B). The ability to sample different conformations may facilitate binding to various partners. Our experiments may suggest that FliS belongs to the family of atypical MG proteins.

The $\alpha$-helical content estimated from the CD spectrum at room temperature is around $51 \%$. This value is in good agreement with that of the homology model subjected to MD simulations. Our studies revealed that moderate concentration of TFE can stabilize the helical structure in FliS increasing its content up to $74 \%$. This observation may also indicate that FliS is not fully folded. Recently Galeva et al. [16] also measured the CD spectrum of FliS while studying its interaction with the flagellar anti-sigma factor FlgM, and reported a significantly larger amount of helical structure in $50 \mathrm{mM}$ Tris- $\mathrm{HCl}$ (pH 7.4) containing $100 \mathrm{mM} \mathrm{NaCl}$. Although our measurements were generally performed without salt in $10 \mathrm{mM}$ Na-phosphate buffer ( $\mathrm{pH}$ 7.4) to allow collection of $\mathrm{CD}$ data below $200 \mathrm{~nm}$, we did not observe any significant salt effect in trial experiments.

FliS is a component of the flagellar export machinery which has several binding partners. Its major function is to serve as an export chaperone for flagellin [2], the main component of bacterial flagellar filaments. It was demonstrated that flagellin binding results in a significant conformational rearrangement within the FliS export chaperone [18]. FliS mediates docking of the FliS-FliC complex to the FlhA component of the export gate situated in the cell membrane $[6,45]$. Through the different binding affinities of various substrate-chaperone complexes, the strictly regulated assembly order of the flagellum may be ensured [6]. FliS also plays a role in the transcriptional regulation of flagellar biosynthesis interacting with the anti-sigma factor FlgM which inhibits the flagellar-specific sigma factor FliA [16,17]. Lam et al. demonstrated that in H. pylori FliS also interacts with co-chaperone HP1076 and with other flagellar associated proteins like FliD and FlgK, displaying general chaperone activity [12]. Various FliS-interacting partners were predicted in the proteome studies of $H$. pylori [13] and C. jejuni [14], suggesting that FliS is a key export chaperone and may be involved in other cellular processes. As FliS has several binding partners within the cell, conformational adaptability seems to be an essential requirement in order to fulfill its various functional roles. A molten globule ensemble of interconverting folded structures may provide a convenient target for conformational selection by diverse partners. 


\section{Acknowledgements}

We thank Professors K. Namba and P. Závodszky for support and encouragement. We are grateful to the Japan Science and Technology Corporation for generous donation of equipment. This work was supported by the Hungarian Scientific Research Fund (OTKA) grants K104726 and NK108642. A.M. and J.K. were supported by the KTIA_NAP_13-22014-0017 program. SRCD experiments were supported by SOLEIL (proposal No. 20140646, 20150515). We thank Frank Wien and Matthieu Réfrégiers at DISCO beamline for their kind help in SRCD data collection. 


\section{References}

1. Minamino, T. (2014) Protein export through the bacterial type III export pathway. Biochim. Biophys. Acta 1843, 1642-1648.

2. Auvray, F., Thomas, J., Fraser, G.M., Hughes, C. (2001) Flagellin polymerisation control by a cytosolic export chaperone. J. Mol. Biol. 308, 221-229.

3. Fraser, G.M., Bennett, J.C., Hughes, C. (1999) Substrate-specific binding of hookassociated proteins by FlgN and FliT, putative chaperones for flagellum assembly. Mol. Microbiol. 32, 569-580.

4. Bennett, J.C.Q., Hughes, C. (2000) From flagellum assembly to virulence: the extended family of type III export chaperones. Trends Microbiol. 8, 202-204.

5. Thomas, J., Stafford, G.P., Hughes, C. (2004) Docking of cytosolic chaperone-substrate complexes at the membrane ATPase during flagellar type III protein export. Proc. Natl. Acad. Sci. USA 101, 3945-3950.

6. Minamino, T., Kinoshita, M., Hara, N., Takeuchi, S., Hida, A., Koya, S., Glenwright, H., Imada, K., Aldridge, P.D., Namba, K. (2012) Interaction of a bacterial flagellar chaperone FlgN with FlhA is required for efficient export of its cognate substrates. Mol. Microbiol. $83,775-788$.

7. Minamino, T., Kinoshita, M., Imada, K., Namba, K. (2012) Interaction between FliI ATPase and a flagellar chaperone FliT during bacterial flagellar protein export. Mol. Microbiol. 83, 168-178.

8. Imada, K., Minamino, T., Kinoshita, M., Furukawa, Y., Namba, K. (2010) Structural insight into the regulatory mechanisms of the flagellar type III chaperone FliT with its binding partners. Proc. Natl. Acad. Sci. USA 107, 8812-8817.

9. Kinoshita, M., Hara, N., Imada, K., Namba, K., Minamino, T. (2013) Interactions of bacterial flagellar chaperone-substrate complexes with FlhA contribute to co-ordinating assembly of the flagellar filament. Mol. Microbiol. 90, 1249-1261.

10. Ozin, A.J., Claret, L., Auvray, F., Hughes, C. (2003) The FliS chaperone selectively binds to the disordered flagellin C-terminal D0 domain central to polymerization. FEMS Microbiol. Lett. 219, 219-224.

11. Muskotál, A., Király, R., Sebestyén, A., Gugolya, Z., Végh, B.M., Vonderviszt, F. (2006) Interaction of FliS flagellar chaperone with flagellin, FEBS Lett. 580, 3916-3920. 
12. Lam, W.W. Woo, E.J., Kotaka, M., Tam, W.K., Leung, Y.C., Ling, T.K., Au, S.W. (2010) Molecular interaction of flagellar export chaperone FliS and cochaperone HP1076 in Helicobacter pylori, FASEB J. 24, 4020-4032.

13. Rain, J.C., Selig, L., DeReuse, V., Battaglia, V., Reverdy, C., Simon, S., Lenzen, G., Petel, F., Wojcik, J., Schachter, V., Chemama, Y., Labigne, A., Legrain, P. (2001) The protein-protein interaction map of Helicobacter pylori. Nature 409, 211-215.

14. Parrish, J.R., Yu, J., Liu, G., Hines, J.A., Chan, J.E., Mangiola, B.A., Zhang, H., Pacifico, S., Fotouhi, F., DiRita, V.J., Ideker, T., Andrews, P., Finley, R.L. Jr. (2007) A proteomewide protein interaction map for Campylobacter jejuni. Genome Biol. 8, R130.

15. Yokoseki, T., Iino, T., Kutsukake, K. (1996) Negative regulation by fliD, fliS, and fliT of the export of the flagellum-specific anti-sigma factor, FlgM, in Salmonella typhimurium. J. Bacteriol. 178, 899-901.

16. Galeva, A., Moroz, N., Yoon, Y.-H., Hughes, K.T., Samatey, F.A., Kostyukova, A.S. (2014) Bacterial Flagellin-Specific Chaperone FliS Interacts with Anti-Sigma Factor FlgM. J. Bacteriol. 196, 1215-1221.

17. Xu, S., Peng, Z., Cui, B., Wang, T., Song, Y., Zhang, L., Wei, G., Wang, Y., Shen, X. (2014) FliS modulates FlgM activity by acting as a non-canonical chaperone to control late flagellar gene expression, motility and biofilm formation in Yersinia pseudotuverculosis. Env. Microbiol. 16, 1090-1104.

18. Evdokimov, A.G., Phan, J., Tropea, J.E., Routzahn, K.M., Peters, H.K., Pokross, M., Waugh, D.S. (2003) Similar modes of polypeptide recognition by export chaperones in flagellar biosynthesis and type III secretion. Nat. Struct. Biol. 10, 789-793.

19. Badger, J., Sauder, J.M., Adams, J.M., Antonysamy, S., Bain, K., Bergseid, M.G., Buchanan, S.G., Buchanan, M.D., Batiyenko, Y., Christopher, J.A., Emtage, S., Eroshkina, A., Feil, I., Furlong, E.B., Gajiwala, K.S., Gao, X., He, D., Hendle, J., Huber, A., Hoda, K., Kearins, P., Kissinger, C., Laubert, B., Lewis, H.A., Lin, J., Loomis, K., Lorimer, D., Louie, G., Maletic, M., Marsh, C.D., Miller, I., Molinari, J., MullerDieckmann, H.J., Newman, J.M., Noland, B.W., Pagarigan, B., Park, F., Peat, T.S., Post, K.W., Radojicic, S., Ramos, A., Romero, R., Rutter, M.E., Sanderson, W.E., Schwinn, K.D., Tresser, J., Winhoven, J., Wright, T.A., Wu, L., Xu, J., Harris, T.J. (2005) Structural analysis of a set of proteins resulting from a bacterial genomics project. Proteins 60, 787-796. 
20. Pfeil, W. (2012) Protein stability and folding: A collection of thermodynamic data. Springer, Berlin, Heidelberg.

21. Sajo, R., Liliom, K., Muskotal, A., Klein, A., Zavodszky, P., Vonderviszt, F., Dobo, J. (2014) Soluble components of the flagellar export apparatus, FliI, FliJ, and FliH, do not deliver flagellin, the major filament protein, from the cytosol to the export gate. Biochim. Biophys. Acta. 1843, 2414-2423.

22. Vonderviszt, F., Kanto, S., Aizawa, S.-I., Namba, K. (1989) Terminal regions of flagellin are disordered in solution. J. Mol. Biol. 209, 127-133.

23. Gasteiger, E., Hoogland, C., Gattiker, A., Duvaud, S., Wilkins, M. R., et al. (2005) Protein identification and analysis tools on the ExPASy server. In: Walker JM editor. The proteomics protocols handbook. Humana Press, NJ. pp. 571-607.

24. Roche, D.B., Buenavista, M.T., Tetchner, S.J., McGuffin, L.J. (2011) The IntFOLD server: an integrated web resource for protein fold recognition, 3D model quality assessment, intrinsic disorder prediction, domain prediction and ligand binding site prediction. Nucleic Acids Res. 39, W171-176.

25. Pronk, S., Páll, S., Schulz, R., Larsson, P., Bjelkmar, P., Apostolov, R., Shirts, M.R., Smith, J.C., Kasson, P.M., van der Spoel, D., Hess, B., Lindahl, E. (2013) GROMACS 4.5: a high-throughput and highly parallel open source molecular simulation toolkit. Bioinformatics 29, 845-854.

26. Aliev, A.E., Kulke, M., Khaneja, H.S., Chudasama, V., Sheppard, T.D., Lanigan, R.M. (2014) Motional timescale predictions by molecular dynamics simulations: case study using proline and hydroxyproline sidechain dynamics. Proteins 82, 195-215.

27. Jorgensen, W.L., Chandrasekhar, J., Madura, J.D., Impey, R.W., Klein, M.L. (1983) Comparison of simple potential functions for simulating liquid water. J. Chem. Phys. 79, 926-935.

28. Kabsch, W., Sander, C. (1983) Dictionary of protein secondary structure: pattern recognition of hydrogen-bonded and geometrical features. Biopolymers 22, 2577-2637.

29. Pettersen, E.F., Goddard, T.D., Huang, C.C., Couch, G.S., Greenblatt, D.M., Meng, E.C., Ferrin, T.E. (2004) UCFS Chimera - A visualization system for exploratory research and analysis. J. Comp. Chem. 25, 1605-1612.

30. Shih, P., Holland, D.R., Kirsch, J.F. (1995) Thermal stability determinants of chicken egg-white lysozyme core mutants: hydrophobicity, packing volume, and conserved buried water molecules. Protein Sci. 4, 2050-2062. 
31. Micsonai, A., Wien, F., Kernya, L., Lee, Y.H., Goto, Y., Réfrégiers, M., Kardos, J. (2015) Accurate secondary structure prediction and fold recognition for circular dichroism spectroscopy. Proc. Natl. Acad. Sci. USA 112, E3995-3103.

32. Cooper, A. (2000) Heat capacity of hydrogen-bonded networks: an alternative view of protein folding thermodynamics. Biophys. Chem. 85, 25-39.

33. Kardos, J., Yamamoto, K., Hasegawa, K., Naiki, H., Goto, Y. Direct measurement of the thermodynamic parameters of amyloid formation by isothermal titration calorimetry. $J$. Biol. Chem. 279, 55308-55314.

34. Munson, M., O’Brien, R., Sturtevant, J.M., Regan, L. (1994) Redesigning the hydrophobic core of a four-helix-bundle protein. Protein Sci. 3, 2015-2022.

35. Schulga, A.A., Makarov, A.A., Levichkin, I.V., Belousova, Y.V., Lobachov, V.M., Protasevich, I.I., Pace, C.N., Kirpichnikov, M.P. (2002) Increased stability of human growth hormone with reduced lactogenic potency. FEBS Letters 528, 257-260.

36. Amadei, A., Marracino, P. (2015) Theoretical-computational modeling of the electric filed effects on protein unfolding thermodynamics. RSC Adv. 5, 96551-96561.

37. Nelson, J.W., Kallenbach, N.R. (1986) Stabilization of the ribonuclease S-peptide $\alpha$-helix by trifluoroethanol. Proteins 1, 211-217.

38. Bruch, M.D., McKnight, C.J., Gierasch, L.M. (1989) Helix formation and stability in a signal sequence. Biochemistry 28, 8544-8561.

39. Baldwin, R.L., Rose, G.D. (2013) Molten globules, entropy-driven conformational change and protein folding. Curr Opin Struct Biol. 23, 4-10.

40. Vamvaca, K., Vögeli, B., Kast, P., Pervushin, K., Hilvert, D. (2004) An enzymatic molten globule: Efficient coupling of folding and catalysis. Proc. Natl. Acad. Sci. USA 101, 12860-12864.

41. Pervushin, K., Vamcava, K., Vogeli, B., Hilvert, D. (2007) Structure and dynamics of a molten globular enzyme. Nat. Struct. Mol. Biol. 14, 1202-1206.

42. Kjaergaard, M., Teilum, K., Poulsen, F.M. (2010) Conformational selection in the molten globule state of the nuclear coactivator binding domain of CBP. Proc. Natl. Acad. Sci USA 107, 12535-12540.

43. Bachmann, A., Wildermann, D., Praetorius, F., Fischer, G., Kiefhaber, T. (2011) Mapping backbone and side-chain interactions in the transition state of a coupled protein folding and binding reaction. Proc. Natl. Acad. Sci. USA 108, 3952-3957. 
44. Mok, K.H., Nagashima, T., Day, I.J., Hore, P.J., Dobson, C.M. (2005) Multiple subsets of side-chain packing in partially folded states of $\alpha$-lactalbumins. Proc. Natl. Acad. Sci. USA 102, 8899-8904.

45. Bange, G., Kümmerer, N., Engel, C., Bozkurt, G., Wild, K., Sinning, I. (2010) FlhA provides the adaptor for coordinated delivery of late flagella building blocks to the type III secretion system. Proc. Natl. Acad. Sci. USA 107, 11295-11300.

46. Willis, B.T.M., Pryor, A.W. (1975) Thermal vibrations in crystallography. Cambridge University Press. 


\section{Figure legends}

\section{Figure 1.}

Temperature induced unfolding of FliS and FliC followed by differential scanning calorimetry. While the calorimetric curve of FliS does not feature any peak corresponding to a cooperative melting transition, the thermal unfolding curve of FliC shows a sharp transition around $48^{\circ} \mathrm{C}$. The curves were corrected by subtracting the buffer baseline. The protein concentration was $0.34 \mathrm{mg} / \mathrm{ml}$ in $10 \mathrm{mM} \mathrm{Na-phosphate} \mathrm{buffer} \mathrm{(} \mathrm{pH}$ 7.4) for both proteins.

\section{Figure 2.}

Thermal unfolding of FliS followed by CD and fluorescence spectroscopy. (A) Series of CD spectra as a function of temperature in the $5-110{ }^{\circ} \mathrm{C}$ range recorded in $7{ }^{\circ} \mathrm{C}$ steps. Arrows show the direction of spectral changes. (B) $\alpha$-helix content of FliS as a function of temperature estimated by the BeStSel method [31]. The overall $\alpha$-helix content is divided into two components by the method. Red circles represent the percentage of residues located at the ends of helices which is proportional to the number of helices and blue squares represent the percentage of residues corresponding to the middle, regular part of $\alpha$-helices. The ratio of the two fractions can be used to estimate the average length of helices. The temperature dependence indicates that, upon heating, first the length of the helices is decreasing and the number of helices starts to decrease at higher temperatures. (C) Heat denaturation of FliS followed by the CD signal at $192 \mathrm{~nm}$. Two, largely overlapping experiments are shown corresponding to scanning rates of 40 (red) and $120{ }^{\circ} \mathrm{C} /$ hour (blue), indicating that the unfolding process reached equilibrium at each temperature point. Dashed line represents a sigmoidal fit regarding a two-state process of $70 \mathrm{~kJ} / \mathrm{mol}$ enthalpy change with a midpoint of $72^{\circ} \mathrm{C}$ and a $\Delta C_{p}$ set to zero. (D) Trp fluorescence excited at $297 \mathrm{~nm}$ as a function of temperature is presented by the $355 / 342 \mathrm{~nm}$ intensity ratio. Linear fittings reveal two phases of heat-induced conformational changes.

\section{Figure 3.}

Demonstration of the folded core and ligand binding ability of FliS. (A) Fluorescence emission spectra of FliS (black), FliC (red) and FliS-FliC complex (green) measured at $15 \mu \mathrm{M}$ protein concentrations. The sum of the FliS and FliC spectra is also presented (blue). The increase of fluorescence intensity and the shift to shorter wavelengths provide evidence for 
complex formation, indicating that the local environment of the single tryptophan residue W122 of FliS became more hydrophobic upon FliC binding. (B) One-dimensional ${ }^{1} \mathrm{H}$ NMR spectrum of FliS $(0.5 \mathrm{mg} / \mathrm{ml})$ in $10 \mathrm{mM}$ Na-phosphate at $\mathrm{pH} 7.4$ and $25{ }^{\circ} \mathrm{C}$ exhibiting welldispersed aromatic/amide proton (6.5-10 ppm) and methyl proton (-0.5-1.5 ppm) regions. Sharp signals at 8.43 and $5.40 \mathrm{ppm}$ arise from the histidine tag. The region between 4.4-5.1 ppm is obscured by residual water signal after presaturation and digital filtering. Chemical shifts were referenced to external 4,4-dimethyl-4-silapentane-1-sulfonate.

\section{Figure 4.}

Limited proteolysis of FliS by (A) trypsin and (B) subtilisin. Proteolytic experiments were done at a protein to protease ratio of $300: 1(\mathrm{w} / \mathrm{w})$. At the indicated time points, portions were removed from the reaction mixture, mixed with electrophoresis sample buffer and boiled for $10 \mathrm{~min}$. Experiments were done in $20 \mathrm{mM} \mathrm{Na} / \mathrm{K}$-phosphate buffer, $150 \mathrm{mM} \mathrm{NaCl}(\mathrm{pH} 7.8)$ at room temperature at $0.4 \mathrm{mg} / \mathrm{ml}$ protein concentration. Intact FliS and the $12 \mathrm{kDa}$ fragment are indicated by arrows.

\section{Figure 5.}

$\alpha$-helix dominated structure of FliS. (A) Salmonella typhimurium FliS homology model was subjected to energy minimization and MD simulation. The final average structure is presented in gradient rainbow colors blue-to-red indicating the N-to C-terminal. W122 side-chain is presented with sticks the environment of which is affected by the N-terminal region of the molecule or the binding substrate of FliS. "Scissors" indicate the proteolytic cleavage sites determined by mass spectrometry. These sites are located at the borders of the high flexibility regions. (B) Flexibility of FliS molecule in the 30-150 ns time interval of the final MD simulation at $300 \mathrm{~K}$ is presented by a tube model. The diameter of the tube reflects the $\beta$ factor values derived from the local fluctuations (RMSF values) upon MD simulation [45]. (C) The distribution of the $\alpha$-helix content upon MD simulations at $300 \mathrm{~K}$ from 6000 snapshots is presented for Salmonella FliS (red), Aquifex FliS (green), lysozyme (blue) and human growth hormone (black). (D) CD spectra of FliS at various TFE concentrations. The measurements were carried out in $10 \mathrm{mM}$ Na-phosphate buffer $(\mathrm{pH} 7.4)$ at a protein concentration of $0.4 \mathrm{mg} / \mathrm{ml}$ at SOLEIL Synchrotron. The originally $51 \% \alpha$-helix content (including the disordered $\mathrm{His}_{6}$-tag) is increased to 59, 68 and $74 \%$ upon addition of 10, 20, 
and 30\% TFE, respectively. Arrows show the direction of spectral changes upon increasing TFE concentration.

\section{Figure 6}

X-ray structures of FliS from different bacterial sources presenting the structural plasticity of the flagellar chaperone. (A) Structure of the monomeric Aquifex aeolicus FliS (PDB: 1ORJ). There are two co-existing conformational variants in the unit cell. The difference is clearly manifested in the N-terminal regions colored blue. One $\mathrm{N}$-terminal is invisible, possibly disordered, while the other one partly forms helical structure and located between the four helices of the bundle, which otherwise can be occupied by the substrate polypeptide chain. (B) The structure of Bacillus subtilis FliS (PDB: 1VH6) forming a dimer of four-helixbundles in a domain swapped manner also shows the structural plasticity of FliS. The two subunits are presented by different colors. 
Figure 1

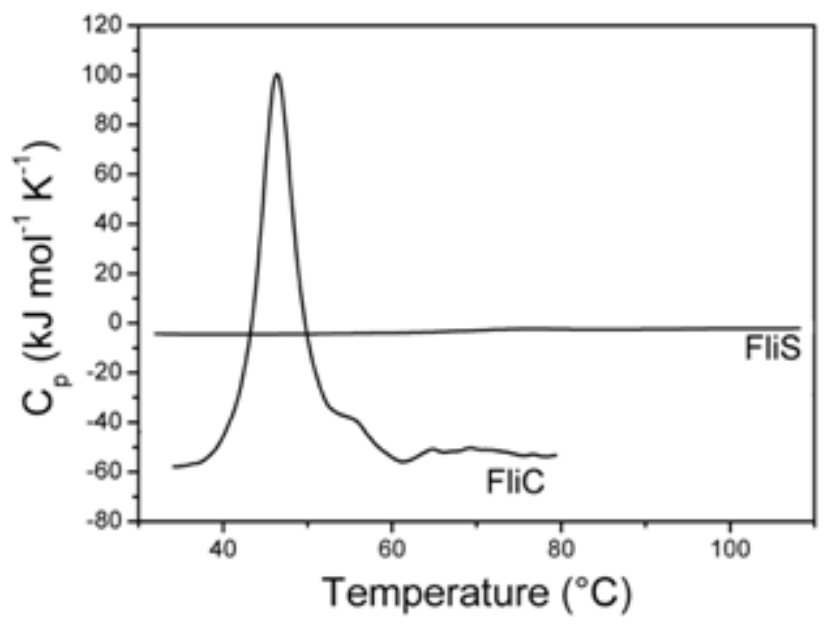


Figure 2
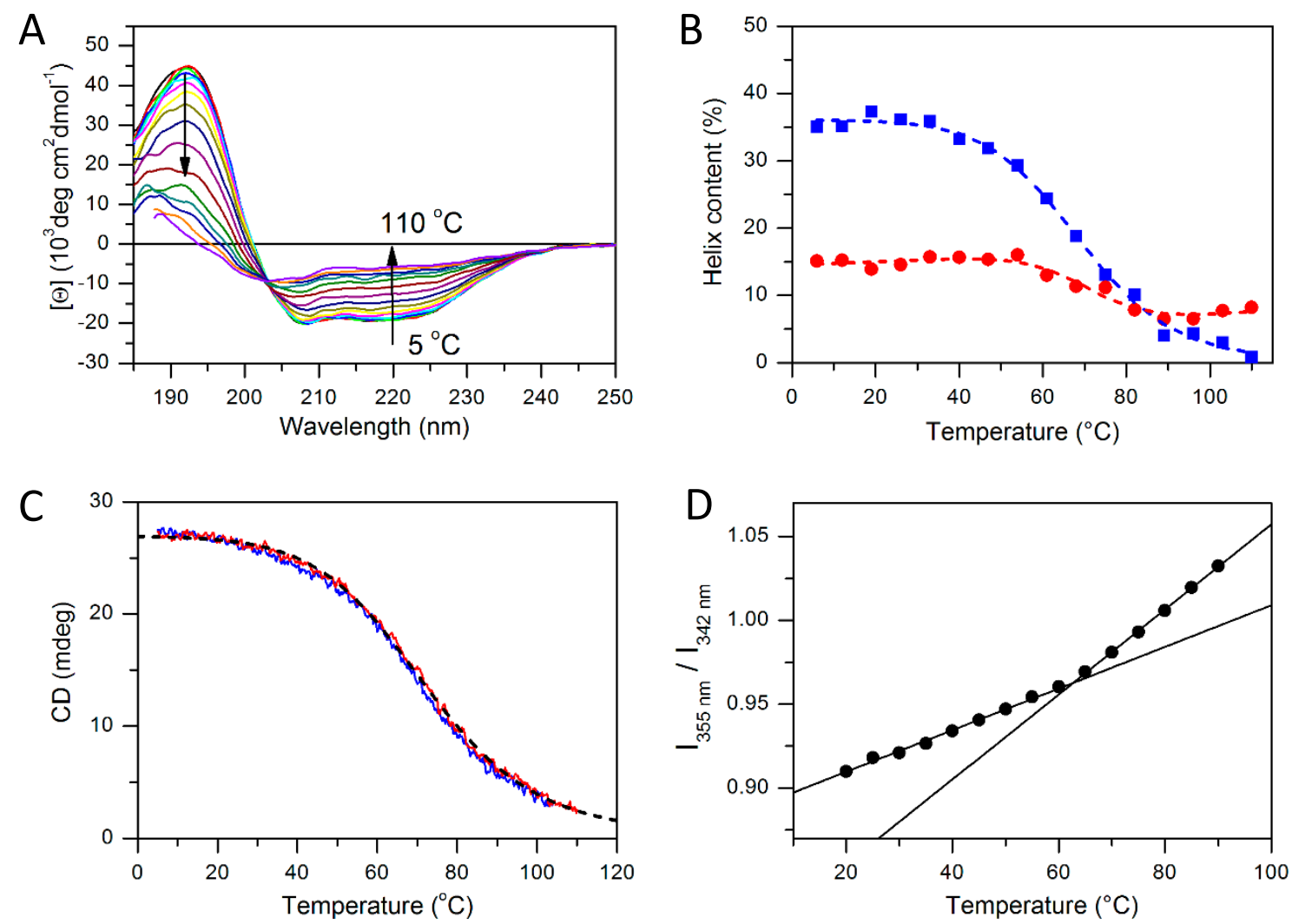
Figure 3

A

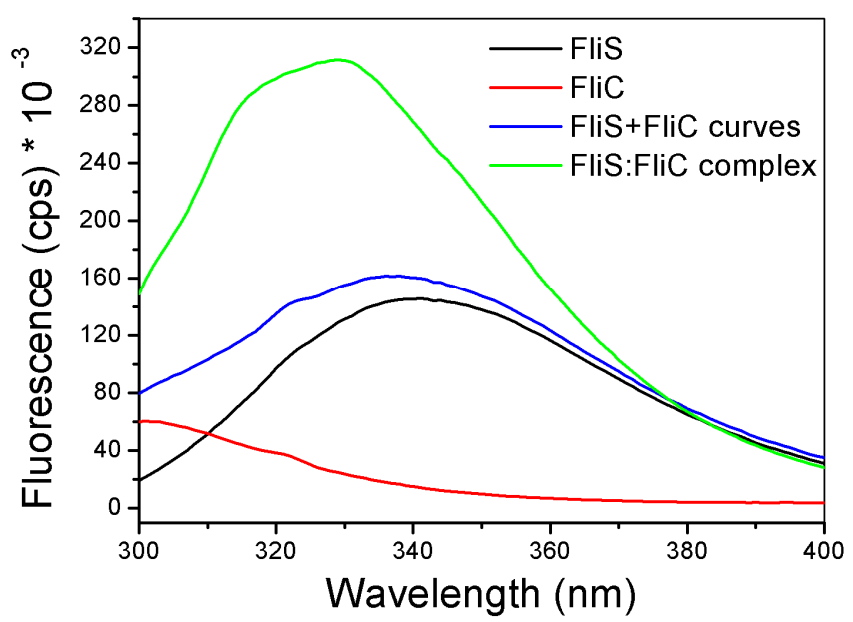

B

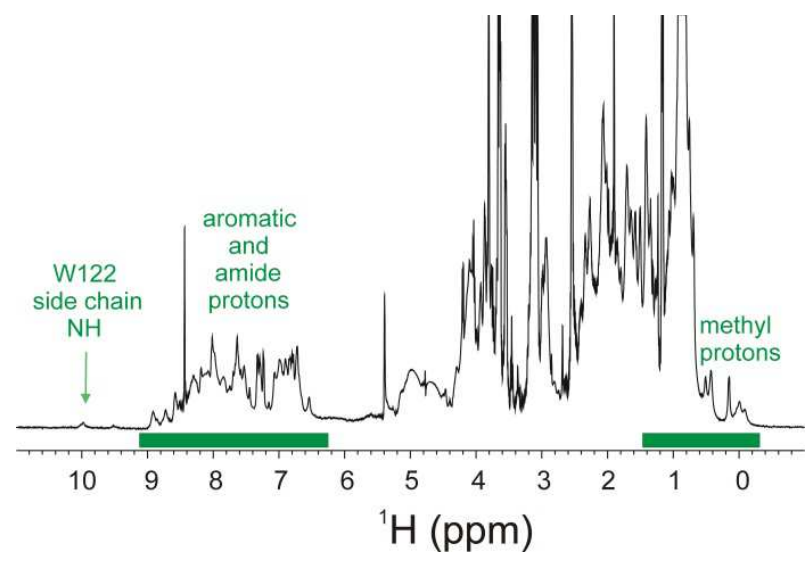


Figure 4

A

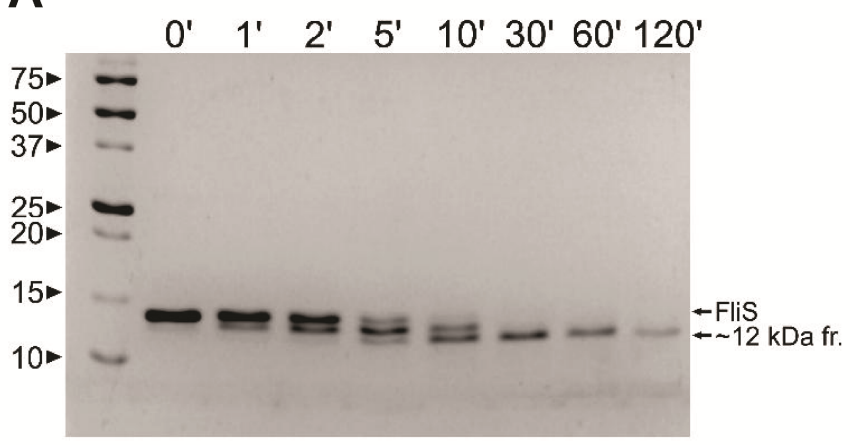

B

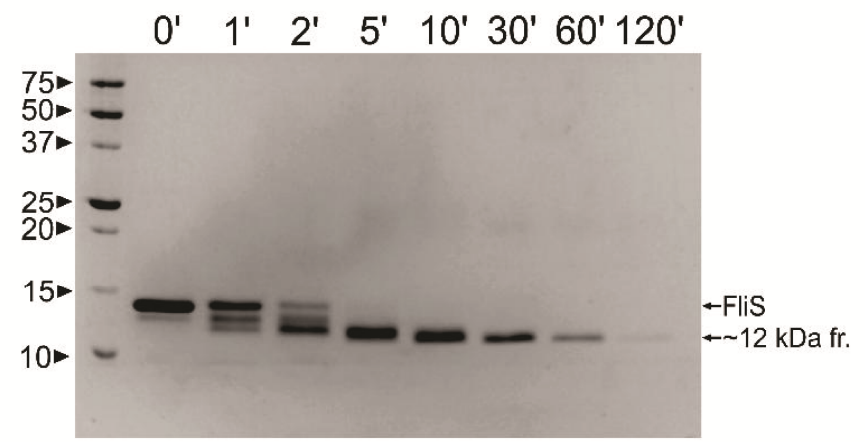


Figure 5
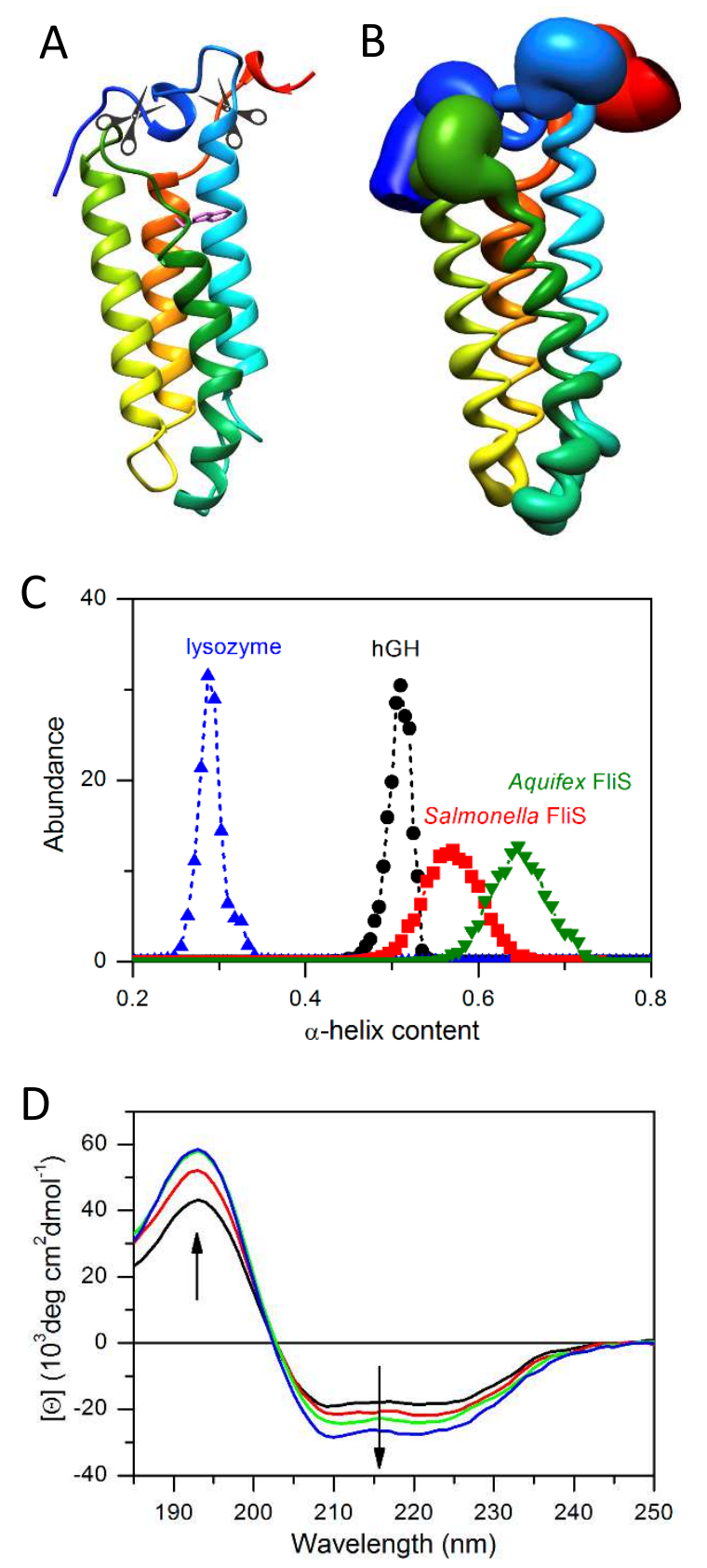
Figure 6

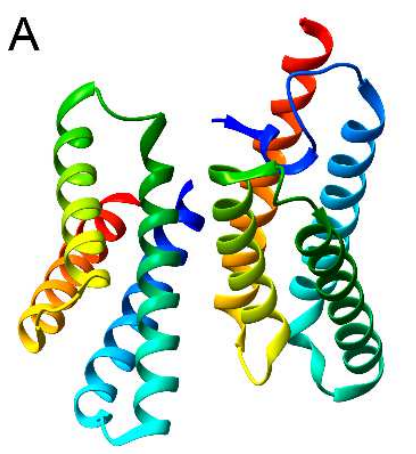

B

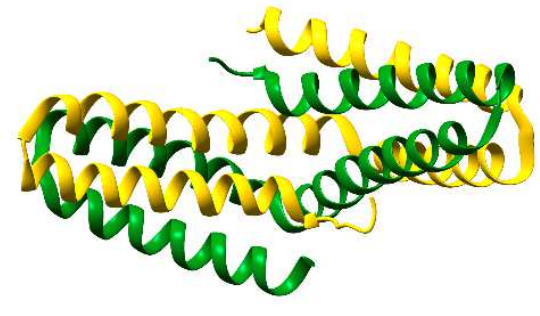

